\title{
DIGITAL SIGNAL PROCESSING IN TRIPLE ANTENNA ARVAS
}

\author{
C.D. Salós ${ }^{\dagger}$, F.M. Lera ${ }^{\ddagger}$, J.L. Villarroel ${ }^{\dagger}$ \\ † Aragón Institute for Engineering Research (I3A) \\ University of Zaragoza \\ Maria de Luna, 1. 50018, Zaragoza, Spain
$\ddagger$ Electronic Engineering and Communications Dpt. University of Zaragoza
Maria de Luna, 1. 50018, Zaragoza, Spain

\begin{abstract}
Time is essential in the rescue of people buried by snow avalanches. An ARVA (French acronym for Appareil de Recherche de Victimes d'Avalanches) is a safety device, which transmits a magnetic field that can be detected by the rescuer's ARVA. Triple antenna ARVAs with DSP (Digital Signal Processor) core have been recently developed to make the search easier and faster, but there are few published studies of the digital signal processing that would give precise results. This paper suggests the different tasks the DSP should perform, i.e. signal filtering, detection and estimation, and proposes several DSP techniques that would give an accurate estimation of the received magnetic field vector.
\end{abstract}

Index Terms - Radio tracking, Safety, Signal processing, Adaptive filters, Signal detection

\section{INTRODUCTION}

The objective of this work is to develop a set of digital signal processing techniques for rescue applications in snow avalaches. An ARVA (Appareil de Recherche de Victimes d'Avalanches), also known as avalanche transceiver or avalanche beacon, is a rescue device used to locate people buried by a snow avalanche. This transceiver generates a pulsed sinusoidal magnetic field of $457 \mathrm{kHz}$ when working in transmission mode. Its characteristics are specified on the standard ETSI 300718. The MF (Medium Frequency) magnetic field signal is not significantly attenuated by the snow, therefore the transmitter can be radiolocated in an area of tens of meters by following the magnetic flux lines. People in the mountain must wear a transmitting ARVA as a safety measure. When people are buried by an avalanche, the rescuers use their ARVAs in reception mode and search through the area covered by the avalanche, trying to detect and track the signal of the victims' transmitter.

ARVAs use solenoid antennas, which consist of a coil with a ferrite core that boosts its sensitivity. The magnetic field produced can be approximated by a magnetic dipole model because of the low frequency used. Thus, the theoretically transmitted field shape is well known and has symmetry of revolution with respect to its axis [1]. Nonetheless, the ferrite core nonlinearities and media discontinuities (earth-snow-air) cause some distortion in the field.

Statistical studies [2] reveal that time is crucial when rescuing people buried by a snow avalanche. The probability of finding alive a victim falls from $93 \%$ within the first 15 minutes of

This work has been funded by the projects DPI2006-07928 (MCYT, Spanish Government) and PM053/2006 (Gobierno de Aragon), and by the agreement between the Gobierno de Aragon and the Universidad de Zaragoza regarding the WALQA research laboratory.

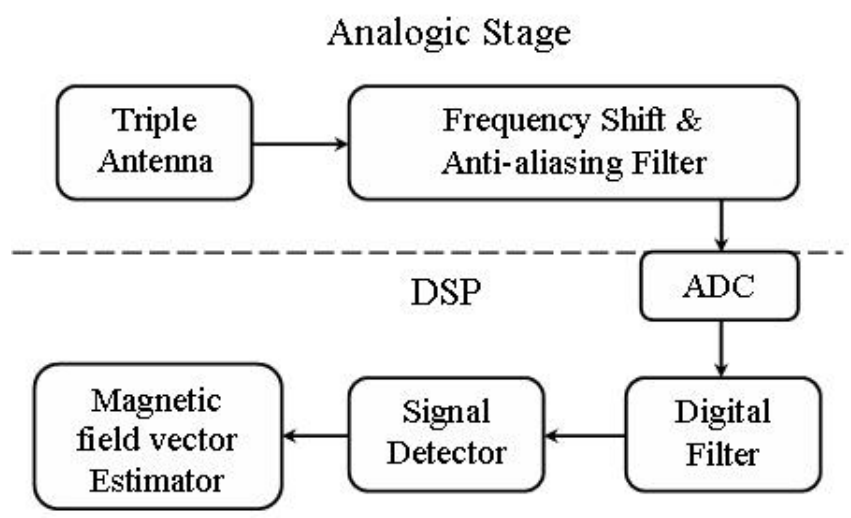

Figure 1. Block diagram of a triple antenna ARVA

the accident ('survival phase') to $23 \%$ if the rescue takes 45 minutes or longer. Because of this, the rescue tasks carried out by the members of the group who have not been buried become essential. However, the signals received are difficult to interpret and require trained people, slowing down the rescue. Recently, a new generation of triple antenna devices has been developed to make easier and speed up the search. Nevertheless, there are few published studies about the digital signal processing techniques that would obtain accurate results even in poor SNR scenarios. The purpose of this paper is to obtain diverse digital signal processing techniques that a triple antenna ARVA could perform in its different tasks, that is, noise filtering, signal detection and, finally, magnitude and direction estimation of the received signal (Figure 1). These DSP techniques result in an accurate estimation of the received magnetic field vector, which could be the base of new automatic searching techniques [3].

The structure of the paper is the following. Section II summarizes the main characteristics of the different ARVA types and Section III provides some general parameters of the problem. Section IV presents some digital filters commonly used in applications that have to deal with the sinusoidal signal cleaning problem and its suitableness to the ARVA scenario, giving special attention to the adaptive one. In Section V different signal detectors in both time and frequency domain are proposed, and the ROC (Receiver Operating Characteristic) curves of their combination with different filters are calculated. Amplitude and phase estimation results are shown in Section VI.

\section{ARVA TYPES}

According to the Faraday's Law, the solenoid antenna presents cosine directivity. Therefore, it will only sense the magnetic field projection over the antenna axis. Noticing that the magnetic field is a three dimensional vectorial quantity, ARVAs with multiple reception antennas have been developed to measure more field components. Only one single transmitting antenna is used. 
The first generation of ARVAs uses one single reception antenna. Made with analogic technology, they only show the received signal power using LEDs or an audible beep. They must be continuously reoriented, looking for the direction of the maximum signal. To solve this problem, digital ARVAs with two perpendicular reception antennas were created. They receive the magnetic field components projected over the plane defined by their coils. As they cannot receive the complete magnetic field, fake maximum signals may appear due to the transmitter orientation and burying depth. These problems slow down the search. The new generation of ARVAs with three mutually orthogonal reception antennas measure the complete magnetic field, magnitude and phase, and process it in a DSP. As the antennas present cosine directivity, the field components sensed by each coil are proportional to the director cosines. Aligning the coils with the Cartesian coordinate's axis, the complete magnetic field magnitude can be easily obtained:

$$
H=\sqrt{H_{x}^{2}+H_{y}^{2}+H_{z}^{2}}
$$

The angle between the magnetic vector and each antenna corresponds to:

$$
\alpha_{i}=\arccos \left(\frac{H_{i}}{H}\right) \text { with } i=x, y, z
$$

To distinguish in which quarter formed by the triple antenna is the arriving signal, 180 phase changes are studied.

A detailed description of ARVA types and search techniques can be found in [4].

\section{GENERAL CONSIDERATIONS}

Previously to the signal processing analysis, there are some aspects that must be borne in mind, such as the characteristics of the noise present at the ADC (Analogue Digital Converter), frequency deviations of the transmitters and the effect of the analogic stage preceding the DSP.

Real noise measurements have been taken from the output of the antenna prototype, showing a lowpass behavior. Its 10th order AR (Auto Regresive) model has been obtained to achieve realistic simulations.

Old analogue transmitters may suffer from frequency drifts due to its age or ambient temperature. These frequency drifts can be greater than the $+/-80 \mathrm{~Hz}$ specified in the standard, causing incompatibility problems with new digital ARVA devices that use narrowband filters as input stage. The solution provided in this paper must be able to deal with frequency deviations up to $+/$ $250 \mathrm{~Hz}$.

The antenna analogic stage determines some general parameters of the next stages performed by the DSP. The prototype uses a triple antenna tuned at $457 \mathrm{kHz}$, followed by an anti-aliasing filter and a mixer that downshifts the received signal to $4 \mathrm{kHz}$. An eight times greater sampling frequency $(32 \mathrm{kHz})$ will be used to assure enough signal samples the DSP will work with.

\section{ARVA SIGNAL FILTERING}

Hostile transmission media, antenna directivity, interferences and noise introduced by the ferrite core degrade the signal, causing a poor SNR. Therefore, a filter is necessary to improve the performance of the following stages. In the problem of filtering a sinusoid, the most popular options are the adapted filter, the bandpass filter and adaptive filtering. To choose the best filter, their joint performance with next stages, i.e. detector and estimator, has to be analyzed. This section only gives the characteristics these filters should have. The adapted filter will not be used because the exact signal frequency is not known as some frequency drift

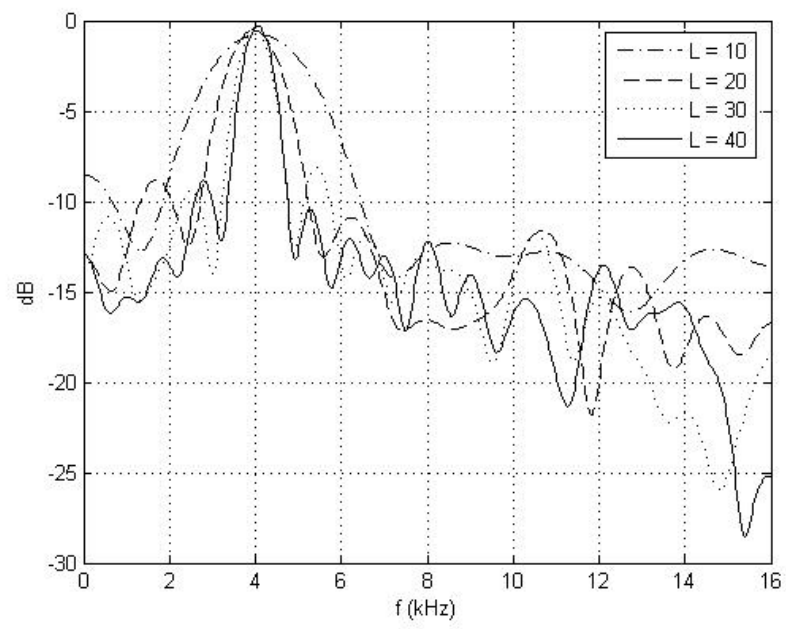

Figure 2. Bandwidth obtained with different filter lengths

is accepted. If both frequencies, the supposed one and the real one, were too different, the filtered signal would be degraded. The bandpass filter under study is a FIR with $50 \mathrm{~dB}$ attenuation outside the range $[3750 \mathrm{~Hz}, 4250 \mathrm{~Hz}]$.

The rest of the section analyzes the adaptive filter that would fit in the application.The ALE (Adaptive Line Enhancer) ([5], [6]) is an adaptive scheme designed to filter a narrowband signal immersed in wideband noise when the signal frequency is unknown, creating its own reference signal as a delayed version of the input.

The LMS (Least Mean Squares) adaptive algorithm has been chosen for its compromise between performance (SNR improvement and convergence speed), robustness and low complexity. The normalized version (NLMS) will be used to avoid convergence problems due to the wide power range of the input signal. It computes the weights $\mathbf{w}$ iteratively using:

$$
\mathbf{w}(n+1)=\mathbf{w}(n)+\frac{\mu}{\delta+\|\mathbf{x}(n)\|^{2}}+\mathbf{x}(n) e^{*}(n)
$$

where $\mu$ denotes the step size, which is related to the rate of convergence and to the final misadjustment. A step size of $\mu=$ 0.1 achieves the compromise. The constant $\delta$ prevents from low power signals and has a typical value of 0.01 .

An analysis of the background noise shows that it is approximately uncorrelated. Thus, the decorrelation delay D of the ALE can be as short as wanted. This delay has not important effects in the behavior of the filter provided that it is long enough to decorrelate the noise. A value of $\mathrm{D}=8$ is taken to prevent from scenarios with correlated noise and to take advantage of the maximum of the correlation of a $4 \mathrm{kHz}$ sinusoid.

The behaviour of the ALE has been analyzed via a simulation series where the input is a $4 \mathrm{kHz}$ ARVA signal (pulsed sinusoid) immersed in coloured noise.

The FIR length is the main parameter that relates bandwidth and complexity. Figure 2 plots the frequency response obtained for different filter lengths $\mathrm{L}$ for a OdB SNR input signal. It is observed that an excessive large length does not necessary result into more effective noise reduction.

Figure 3 shows the evolution of the adaptive filter frequency response along the time in high and poor SNR scenarios. One of the most important characteristics is the elimination of the passband in case of signal absence if the SNR is not too high (Figure 3 a). This fact will improve the signal pulse detection rate.

In high SNR scenarios, residual passbands appear even in signal absence after a pulse, as well as secondary passbands at 


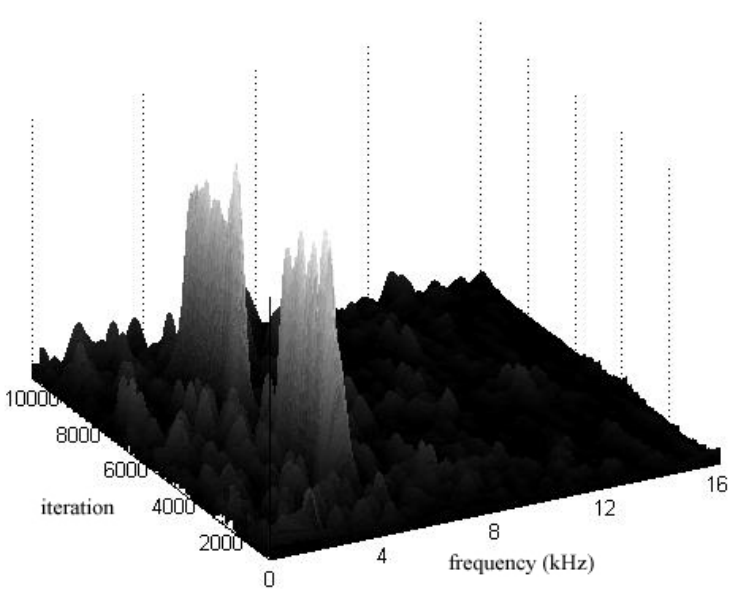

(a) $\mathrm{SNR}=0 \mathrm{~dB}$

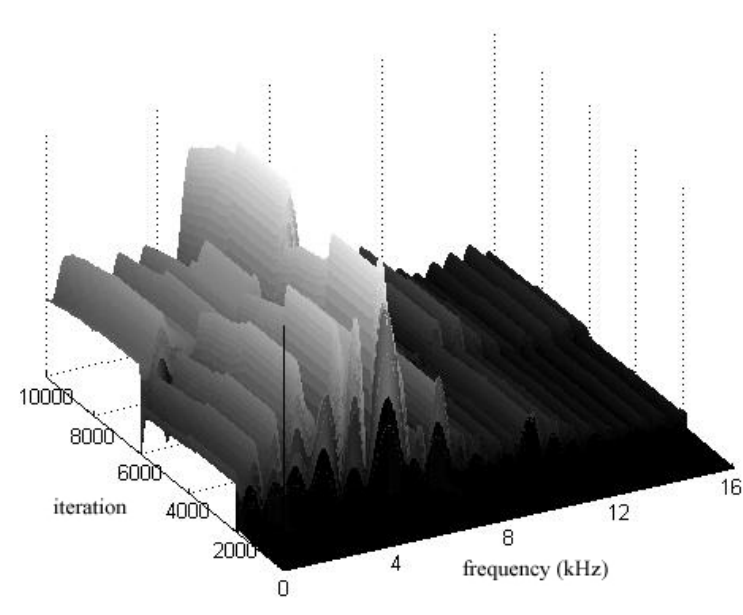

(b) $\mathrm{SNR}=40 \mathrm{~dB}$

Figure 3. ALE frequency response evolution. The desired signal pulses are in the samples 2000-4000 and 6000-8000.

those frequencies where the desired signal presents correlation maximums, specially at the half and the double of the signal frequency (Figure $3 \mathrm{~b}$ ). These undesired residual passbands do not represent a problem because they only appear in good SNR conditions, when the noise power is not high enough to disturb significantly the filter coefficients.

\section{ARVA SIGNAL DETECTION}

To save batteries, ARVAs transmit a pulsed signal. Because of this, a detector is necessary to distinguish when a signal pulse is present. The lower the SNR at which the detector can work, the longer will be the available search range.

The main block of a detector calculates some signal parameter, usually power, and compares it with a threshold. The time domain power estimation and the periodogram have been studied. The advantage of the periodogram is that it presents the signal power in frequency terms, allowing to focus the search in the frequency range of interest, in our case $[3750 \mathrm{~Hz}, 4250 \mathrm{~Hz}$, eliminating noise from other frequency bands. The FFT length is related to frequency and time accuracy, as it happens in a spectrogram. A long FFT provides more frequency bins, but increases the time uncertainty in the pulse detection. Although with a sampling frequency of $32 \mathrm{kHz}$ a $250 \mathrm{~Hz}$ resolution requires a 128

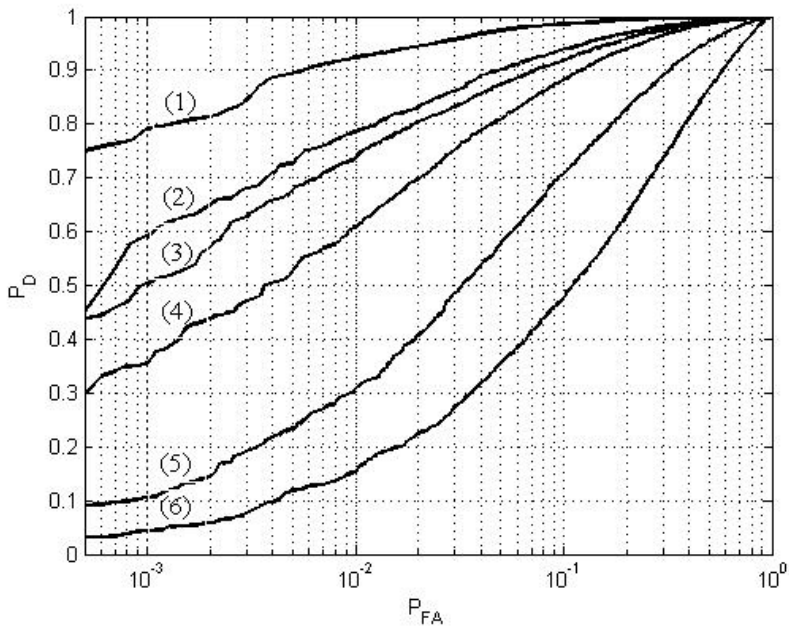

Figure 4. ROC curves for the following combinations: (1) ALE \& periodogram, (2) ALE \& time power estimation, (3) nonadaptive filter \& periodogram, (4) non-adaptive filter \& time power estimation, (5) no filter \& periodogram, (6) no filter \& time power estimation

point FFT, shorter lengths will also be studied trying to achieve a more accurate control of the pulse limits.

The effect of previous filtering to the detector is also analized. The $[3750 \mathrm{~Hz}-4250 \mathrm{~Hz}]$ passband filter and the ALE with NLMS obtained in the previous section have been used.

To compare the detection results of each system, the ROC (Receiver Operating Characteristics) curves of the different filter and detector combinations have been obtained. The ROC plots the detection probability $P_{D}$ against the false alarm probability $P_{F A}$. They have been calculated from a set of simulations for different SNR situations, following a method based on the one proposed in [7]. Figure 4 shows the ROC curves of different detector systems with a 64 point FFT, a 32 coefficients ALE and an SNR of $-6 \mathrm{~dB}$.

The results without a filter previous to the detector are the poorest ones. The adaptive filter ALE gives much lower false alarm rates than the non-adaptive filter because it eliminates the passband when there is no input signal.

The periodogram is the detector that presents better ROC curves, because it eliminates the noise power from non-interest frequency bands. With a $32 \mathrm{kHz}$ sampling frequency, a 128 point FFT is necessary to obtain the desired $500 \mathrm{~Hz}$ range. Undesired noise is not eliminated with shorter FFTs, and the results with more frequency bins do not present a special improvement.

Once the most suitable filter (ALE) and detector (128 point FFT periodogram) have been selected, the most appropriate FIR length has to be chosen. The higher is the filter order, the narrower will be its passband, obtaining better ROC curves at the expense of higher computational costs. A 48 coefficients filter is sufficient to obtain a reliable detector $\left(P_{D}=0.97\right.$ and $P_{F A}=10^{-3}$ with $S N R=-6 d B$ ).

\section{SIGNAL PARAMETERS ESTIMATION}

The final objective of this work is to obtain an accurate magnetic field vector measurement. Its magnitude and direction are calculated with the amplitude and phase estimated from the signal received by each antenna. The MLE (Maximum Likelihood Estimator) and LSE (Least Squares Estimator) estimators are studied. In the case of sinusoid frequency, amplitude and phase estimations, they result in the same formulas and their derivation can 
be found in [8]. It is known that the MLE is asymptotically unbiased and efficient, that is, it approximates the optimum MVU (Minimum Variance Unbiased) estimator when a high enough number of samples are used.

To analyze the estimation quality, its bias was studied and its variance is compared with the Cramer-Rao Lower Bound (CRLB), calculated under the assumption of additive white gaussian noise. The effect of adaptive and non-adaptive filtering previously to the estimator was also studied.

According to the standard ETSI 300718, the minimum pulse width is of $70 \mathrm{~ms}$, resulting into a maximum of 2240 signal samples we can assume the DSP can work with. Actually, the number of samples is lower because of some time inaccuracy of the pulse detector.

The frequency MLE is obtained by maximizing the periodogram. As the frequency is limited to $[3750 \mathrm{~Hz}, 4250 \mathrm{~Hz}$, the periodogram obtained via FFT can be substituted by a Chirp$\mathrm{Z}$ transform (CZT) focused in the interest frequency range, increasing the precision in the estimation.

A $0.49 \mathrm{~Hz}$ resolution offered by a 1024-point CZT is enough for the application. Simulation results show that as the number of signal samples $\mathrm{N}$ used in the estimation increases, the bias is reduced. Although the variance decreases as the SNR or N increases, it is still much bigger than the CRLB. A number of $\mathrm{N}$ $=650$ samples achieves accurate estimations. It is also observed that he ALE filter slightly reduces the bias.

Approximate amplitude and phase MLEs can be found if $f_{0}$ is not near 0 or the Nyquist frequency, where $\hat{f}_{0}$ is obtained with the CZT estimator:

$$
\begin{gathered}
\hat{A}=\frac{2}{N}\left|\sum_{n=0}^{N-1} x(n) \exp \left(-j 2 \pi \hat{f}_{0}\right)\right| \\
\hat{\phi}=\arctan \left(-\frac{\sum_{n=0}^{N-1} x(n) \sin \left(2 \pi \hat{f}_{0} n\right)}{\sum_{n=0}^{N-1} x(n) \cos \left(2 \pi \hat{f}_{0} n\right)}\right)
\end{gathered}
$$

A simulation series has proved that, in the amplitude MLE, the bias error decreases as $\mathrm{N}$ or the SNR increase. A number of samples of $\mathrm{N}=1000$ produces small enough variance and bias errors. The closeness of the variance obtained to the CRLB and the small bias values obtained verify the estimator quality.

Neither previous ALE filtering, due to its SNR dependent scaling factor, nor non-adaptive filters (adapted filter included) provide better estimation results, so in this case the signal will not be previously filtered.

The variance of the phase MLE follows the CRLB, but the bias shows a behaviour independent from the SNR or N. Therefore, the desired variance will be the parameter that determines the number of signal samples to use. Noticing that expanding the complex exponential of (4) in sines and cosines gives the same multiplications as in (5), the same $\mathrm{N}$ can be used, although such phase precision is not necessary. It has been also verified that a previous filter does not achieve any improvement. Tables 1-3 summarize the results for a signal frequency of $4 \mathrm{KHz}$ and unity amplitude.

Table 1. Frequency estimation.

\begin{tabular}{|c||c|c|c|c|c|}
\hline & $-10 \mathrm{~dB}$ & $-5 \mathrm{~dB}$ & $0 \mathrm{~dB}$ & $5 \mathrm{~dB}$ & $10 \mathrm{~dB}$ \\
\hline \hline bias & $3 \cdot 10^{-1}$ & $7 \cdot 10^{-2}$ & $6 \cdot 10^{-2}$ & $4 \cdot 10^{-2}$ & $2 \cdot 10^{-2}$ \\
\hline$\sigma^{2}$ & 12.2 & 3.2 & 2.8 & 0.8 & 0.2 \\
\hline CRLB & $3 \cdot 10^{-6}$ & $10^{-6}$ & $3 \cdot 10^{-7}$ & $10^{-7}$ & $3 \cdot 10^{-8}$ \\
\hline
\end{tabular}

Table 2. Amplitude estimation.

\begin{tabular}{|c||c|c|c|c|c|}
\hline & $-10 \mathrm{~dB}$ & $-5 \mathrm{~dB}$ & $0 \mathrm{~dB}$ & $5 \mathrm{~dB}$ & $10 \mathrm{~dB}$ \\
\hline \hline bias & $2 \cdot 10^{-2}$ & $9 \cdot 10^{-3}$ & $3 \cdot 10^{-3}$ & $2 \cdot 10^{-3}$ & $10^{-3}$ \\
\hline$\sigma^{2}$ & $10^{-2}$ & $5 \cdot 10^{-3}$ & $2 \cdot 10^{-3}$ & $5 \cdot 10^{-4}$ & $2 \cdot 10^{-4}$ \\
\hline CRLB & $10^{-2}$ & $3 \cdot 10^{-3}$ & $10^{-3}$ & $3 \cdot 10^{-4}$ & $10^{-4}$ \\
\hline
\end{tabular}

Table 3. Phase estimation.

\begin{tabular}{|c||c|c|c|c|c|}
\hline & $-10 \mathrm{~dB}$ & $-5 \mathrm{~dB}$ & $0 \mathrm{~dB}$ & $5 \mathrm{~dB}$ & $10 \mathrm{~dB}$ \\
\hline \hline bias & $3 \cdot 10^{-2}$ & $10^{-2}$ & $6 \cdot 10^{-3}$ & $5 \cdot 10^{-3}$ & $5 \cdot 10^{-3}$ \\
\hline$\sigma^{2}$ & $6 \cdot 10^{-2}$ & $2 \cdot 10^{-2}$ & $6 \cdot 10^{-3}$ & $2 \cdot 10^{-3}$ & $10^{-3}$ \\
\hline CRLB & $4 \cdot 10^{-2}$ & $10^{-2}$ & $4 \cdot 10^{-3}$ & $10^{-3}$ & $4 \cdot 10^{-4}$ \\
\hline
\end{tabular}

\section{CONCLUSIONS}

The different blocks of a triple antenna ARVA DSP have been defined. An adaptive filter is preferred, specially previously to the signal detector, because it creates a passband only during the signal pulses. A frequency domain signal detector offers high $P_{D}$ and low $P_{F A}$ ROC curves even under poor SNR, focusing the search in the desired frequency range. Maximum likelihood estimators have shown to approximate their respective MVU estimator, resulting in an accurate received magnetic field vector measurement. The different signal processing blocks have been implemented in a Texas Instruments DCS (Digital Signal Controller) TMS320F2812, obtaining direction errors from the order of hundredths of radian.

The magnetic field vector obtained in this work will be the base of new localization techniques. Future work will also include the study of the multiple burial scenario, where signal processing techniques will be used to distinguish between the different received signals. The earth-snow-air discontinuity effects on the magnetic field shape and in the different search methods will also be analized.

\section{REFERENCES}

[1] D.K. Cheng,Field and Wave Electromagnetics, Adisson Wessley.

[2] M. Falk, H. Brugger, L. Kastner,"Avalanche survival chances," The Newsletter of the International Society for Mountain Medicine, vol. 4, 1994.

[3] P. Piniés, J.D. Tardós, "Fast localization of avalanche victims using Sum of Gaussians," in Proc. IEEE International Conference in Robotics and Automation, Orlando, May 2006, pp. 3989-3994.

[4] A. Ayora, A. Casals, J. Villarroel, J. Tardós, "Rescates en avalanchas," Desnivel, vol. 221, 2005, pp. 72-78 (in Spanish).

[5] S. Haykin,Adaptive Filter Theory, Prentice Hall Information and System Sciences Series.

[6] A.K. Sayed,Fundamentals of Adaptive Filtering, John Wiley \& Sons Inc.

[7] E.K. Hung, R.W. Herring, "Simulation Experiments to Compare the Signal Detection Properties of DFT and MEM Spectra," IEEE transactions on acoustics, speech, and signal processing, vol. 29, no. 3, pp. 1084-1089, Oct. 1981.

[8] S.M. Kay, Fundamentals of Statistical Signal Processing: Estimation Theory, Prentice Hall Signal Processing Series. 